\title{
Hydrodynamic conditions in laser irradiated buried layer experiments
}

Cite as: Phys. Plasmas 27, 063301 (2020); https://doi.org/10.1063/5.0004506

Submitted: 12 February 2020 . Accepted: 12 May 2020 . Published Online: 02 June 2020

Yechiel Frank (D), Gregory E. Kemp, Edward V. Marley, Gabriel Pérez Callejo (D), Mark E. Foord, Marilyn B. Schneider (D), Yosi Ehrlich (D), and Moshe Fraenkel

ARTICLES YOU MAY BE INTERESTED IN

An analytic asymmetric-piston model for the impact of mode-1 shell asymmetry on ICF implosions

Physics of Plasmas 27, 062704 (2020); https://doi.org/10.1063/5.0001335

Hotspot conditions achieved in inertial confinement fusion experiments on the National Ignition Facility

Physics of Plasmas 27, 050901 (2020); https://doi.org/10.1063/5.0003298

Theory of ignition and burn propagation in inertial fusion implosions

Physics of Plasmas 27, 052708 (2020); https://doi.org/10.1063/1.5143889 


\title{
Hydrodynamic conditions in laser irradiated buried layer experiments
}

\author{
Cite as: Phys. Plasmas 27, 063301 (2020); doi: 10.1063/5.0004506 \\ Submitted: 12 February 2020 . Accepted: 12 May 2020 . \\ Published Online: 2 June 2020
}

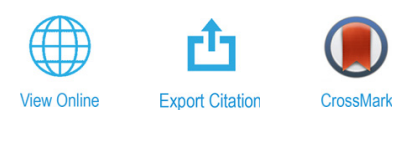

Yechiel Frank, ${ }^{7, a)}$ (D) Gregory E. Kemp, ' Edward V. Marley, 'Gabriel Pérez Callejo, ${ }^{2}$ (D) Mark E. Foord, Marilyn B. Schneider, (D) Yosi Ehrlich, ${ }^{3}$ (D) and Moshe Fraenkel ${ }^{3}$

\author{
AFFILIATIONS \\ 'Lawrence Livermore National Laboratory, 7000 East Ave., Livermore, California 94550, USA \\ ${ }^{2}$ Department of Physics, Clarendon Laboratory, University of Oxford, Parks Road, Oxford OX1 3PU, United Kingdom \\ ${ }^{3}$ Plasma Physics Department, Soreq NRC, Yavne 81800, Israel
}

a) Author to whom correspondence should be addressed: frank28@llnl.gov

\begin{abstract}
The calculation of open shell ionization level and radiative properties of materials in Non-Local Thermal Equilibrium (NLTE) is currently still a major challenge for any atomic model. The predictions of various NLTE atomic codes at these conditions still differ significantly. In recent years, a new buried layer platform was developed at the Lawrence Livermore National Laboratory and the Laboratory for Laser Energetics. This platform is used to measure ionization distribution and emission of open L-shell, mid-Z ions and open M-shell, high-Z ions at NLTE conditions that are relevant in many laser plasma applications. These experiments offer a unique chance for benchmarking the atomic models. In order to perform these experiments, a uniform well characterized plasma source is required. In this work, we present onedimensional (1D) and two-dimensional simulations of the experimental platform. These simulations were used for both the design and the analysis of the experiments. The simulations demonstrate the different phases of hydrodynamic evolution of the target and identify the time windows in which uniform conditions can be achieved. A 1D expansion of the target was found to be adequate to describe the target's evolution for most of the experiment duration. The fast $1 \mathrm{D}$ simulations were compared with recent experimental results from the Omega laser facility. The sensitivity of the results to several modeling parameters such as the electron flux limiter and laser resonant absorption is reported.
\end{abstract}

Published under license by AIP Publishing. https://doi.org/10.1063/5.0004506

\section{INTRODUCTION}

Non-local thermodynamic equilibrium (NLTE) radiative processes, such as emission and absorption of radiation, play an important role in studying a wide range of laboratory and astrophysical plasmas. ${ }^{1}$ In recent years, vast progress has been made in the development of new laboratory plasma sources, from the National Ignition Facility (NIF) to the future Extreme Light Infrastructure project (ELI), powerful Z-pinch machines, and X-ray free electron lasers. This progress increases the demand for new, fast, and accurate modeling of plasmas. For example, during a typical Inertial Confinement Fusion (ICF) simulation, the laser blow-off plasma requires NLTE atomic physics modeling over a wide range of densities and temperatures. The main challenge in modeling NLTE plasmas is that the number of level populations that can be calculated with even modern machines is not much larger than $\sim 10^{6}$ levels. $^{2}$ However, for many applications particularly in medium to high $\mathrm{Z}$ hot plasmas, even this number is not sufficient.
In these cases, the computing power required for detailed level accounting calculations is overwhelming. Therefore, models that take into account average atomic quantities are commonly used for practical calculations. Developing an experimental platform that can provide uniform well diagnosed plasma conditions is crucial for benchmarking and improving the NLTE atomic models.

A buried layer platform has been developed in recent years at Lawrence Livermore National Laboratory (LLNL) and Laboratory for Laser Energetics (LLE) for this purpose. ${ }^{3-5}$ The experimental results were presented in previous publications, and some aspects of the simulations were reported in Ref. 4. However, analysis of the evolution of plasma conditions in these targets, focusing on the expansion dynamics, was not yet presented.

In this work, we present one-dimensional (1D) and twodimensional (2D) radiation-hydrodynamics (rad-hydro) simulations of these experiments. These simulations are important for two reasons: 

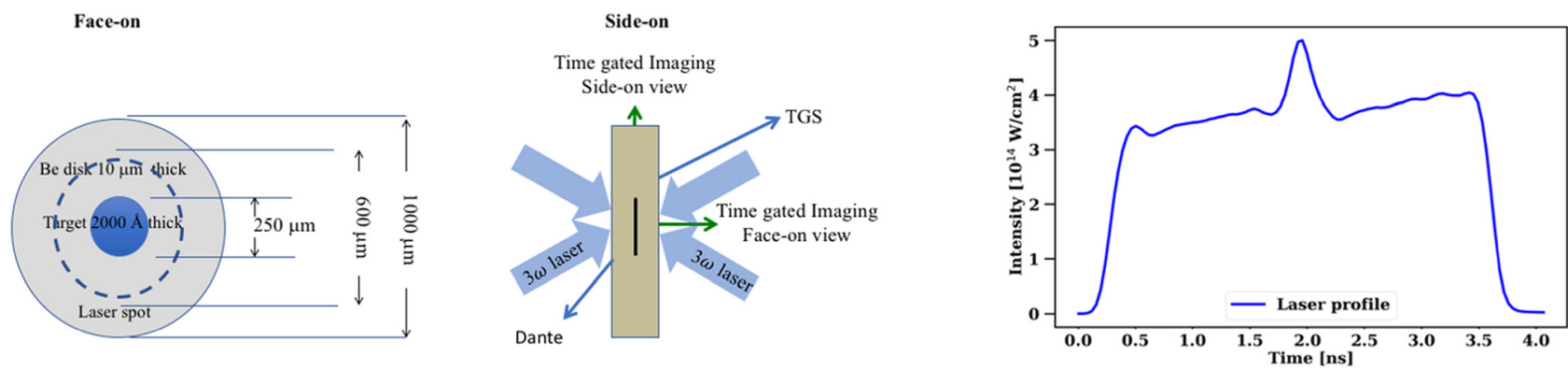

FIG. 1. Experimental setup. Left figure shows a face-on view of the target. The solid blue circle is the buried layer target. The dashed circle represents the laser spot coverage area. The large gray circle is the total size of the Be disk. Middle figure shows a side-on view of the target and the various diagnostics used. Additional high resolution spectrometers were fielded, measuring the emission in specific photon energy ranges during the experiments. However, these measurements are not discussed in this paper. Right figure shows the temporal intensity profile of the laser pulse on each side of the target.

First, they show that a $1 \mathrm{D}$ description is indeed adequate to describe the axial expansion of the target for most of the experiment duration. Second, these simulations are an important tool in order to identify the time frames in which uniform plasma conditions can be achieved. The actual plasma conditions are experimentally measured using imaging and spectroscopic techniques. However, in designing the experiments, these simulations can give an initial evaluation of the plasma conditions achievable at a given experimental configuration.

This paper is organized in the following way: In Sec. II, the experimental setup is briefly described and experimental data are presented. In Sec. III, the radiation-hydrodynamics and atomic physics codes used for this work are described. Section IV presents the time evolution of plasma parameters in the target. Results of 1D and 2D simulations are compared and discussed. In Sec. V, some features of the simulations are compared with the experimental results. In Sec. VI, the sensitivity of the simulations to various parameters is presented. Finally, we conclude our findings in Sec. VII.

\section{EXPERIMENTAL SETUP}

The experimental setup was previously described in several papers. ${ }^{3-5}$ In this section, we describe target and laser conditions relevant for this work. The target consists of a $1000 \mu \mathrm{m}$ diameter, $5 \mu \mathrm{m}$ thick beryllium disk. A $250 \mu \mathrm{m}$ diameter sample target was coated onto the Be disk. A final $5 \mu \mathrm{m}$ over-coating of Be is then added to the target. Several different types of buried samples were used during the experiments. In this work, we will focus on two target configurations. The first type of targets consisted of $1000 \AA$ Fe and $1000 \AA$ of V, comixed. The second type targets consisted of $2000 \AA$ of Ti only.

The Be layers act as inertial tampers to slow the expansion of the sample both in the radial and the axial directions. In previous experiments, where a smaller Be disk diameter was used, radial confinement was not achieved throughout the experiment. ${ }^{4}$ The sample is laser heated on each side by 18 beams with an intensity of $\approx 3-4 \times 10^{14} \mathrm{~W} / \mathrm{cm}^{2}$ per side. The intensity of the laser spot rolls off to $90 \%$ at a diameter of $600 \mu \mathrm{m}$ ensuring uniform heating of the buried sample. A schematic representation of the experimental setup is presented in Fig. 1.

Two pinhole cameras were coupled to framing cameras to image the time-resolved emitting volume of the plasma. One camera viewed the target face-on to measure the radial dimension of the plasma and the second viewed the target side-on to measure the axial expansion of the plasma. The pinhole cameras had a magnification of
$6 \times$ and a spatial resolution of $\approx 20 \mu \mathrm{m}$. An example of the raw imaging data can be seen in Fig. 2. In Sec. V, when comparing experimental axial expansion of the target to the simulated one, an average value over the radial dimension was used.

Two broad-band X-ray spectrometers were fielded in some experiments. Dante, an array of 18 channels of $\mathrm{x}$-ray diodes coupled to mirrors and filters, measures time-dependent emission spectrally integrated within those channels. ${ }^{6}$ A Transmission Grating Spectrometer (TGS) developed at the Soreq research center Israel was fielded in some of the experiments. The TGS gives a more spectrally detailed measurement with $\Lambda / \Delta \Lambda=10-20$ (wavelength dependent) over the $200-3000 \mathrm{eV}$ photon energy range range. The TGS was coupled to a CCD camera resulting in time-integrated measurements only. The TGS and Dante diagnostics are each independently calibrated. In addition to the diagnostics shown in Fig. 1, several higher resolution spectrometers were fielded to measure the emission in specific photon

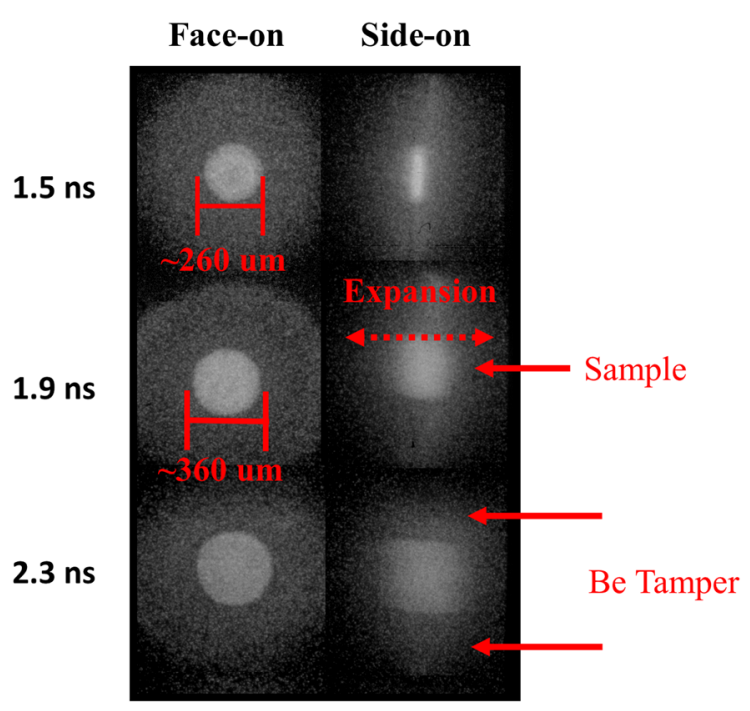

FIG. 2. An example of the target imaging results. Shown are face-on and side-on images at three times during the experiments. Bright emission region at the center is the buried target, surrounding halo is the Be tamper. The full data set consists of four frames in each time strip. The four frames are taken over a period of $200 \mathrm{ps}$. 
energy ranges during the experiments. However, these measurements are not discussed in this work.

\section{SIMULATION CODES}

Several simulation codes were used in this work. 1D simulations were performed using the planar Lagrangian code, Florence, ${ }^{8}$ which uses NLTE tabulated atomic properties calculated with the atomic code SEMILLAC. ${ }^{9,10}$ 2D simulations were performed using the code Hydra, ${ }^{11}$ using in-line NLTE calculated atomic properties from the DCA code. ${ }^{2}$

Both rad-hydro codes simulate the hydrodynamic evolution together with multi-group radiation transport in the plasma. Laser energy deposition is described using an inverse bremsstrahlung model, with the assumption that a given fraction of the energy not absorbed during propagation toward the critical density surface is absorbed at the critical density surface and the rest is back reflected and continues to be absorbed on its way back. Electron heat conductivity is described by the Spitzer model ${ }^{12}$ combined with a flux limiter.

A sensitivity test of the results to the choice of the laser absorption at critical density parameter and flux limiter value is presented in Sec. VI.

The main differences between the two codes besides the dimensionality are the different atomic models, the use of tabulated opacities data vs in-line calculation, and the use of the diffusion approximation in Florence and a full transport solution in HYDRA. As discussed in Sec. IV, the different radiation transport models can be the cause of differences in early time temperature dynamics (as seen in Fig. 4). However, since other hydrodynamic parameters were kept the same in both simulations, the main difference in the duration of the burnthrough phase and terminal temperature is likely to be caused primarily by the dimensionality of the codes.

In both $1 \mathrm{D}$ and $2 \mathrm{D}$ simulations, the experimental laser profile was used. In the $1 \mathrm{D}$ case, this manifests in the temporal shape only while in the $2 \mathrm{D}$ case, it is reflected in the spatial profile as well. In the $1 \mathrm{D}$ calculations presented in this work, a symmetry around $\mathrm{z}=0$ is used. While the laser profiles on different sides of the target had minor temporal and spatial differences, no major effect of these differences on the target's symmetry was observed in the $2 \mathrm{D}$ simulations or in the experimental results.

\section{DYNAMIC EVOLUTION OF PLASMA CONDITIONS}

In order to identify the time frames in which approximately uniform and stationary plasma conditions can be achieved, we examine the time evolution of the plasma conditions at several locations marked as a, b, and c in Fig. 3 .

The temperature and density time evolution at the center of the target, $Z=0, r=0$, marked as point a in Fig. 3, are presented in Fig. 4. Dashed lines are the $2 \mathrm{D}$ simulations results. Solid lines are the $1 \mathrm{D}$ results. Good agreement is found between the two simulations for times $\leqslant 2 \mathrm{~ns}$. At later times, the qualitative behavior of the two simulations remains similar. However, the 2D simulation shows slower dynamics and achieves steady-state at lower temperature and density.

The target evolution can be divided into four phases:

1. During the first $\sim 0.5 \mathrm{~ns}$, a shock from the impact of the laser on the tamper layers is traveling back and forth in the target several times. At this phase, the material temperature reaches a few $10 \mathrm{~s}$ of $\mathrm{eV}$ and density is near solid.

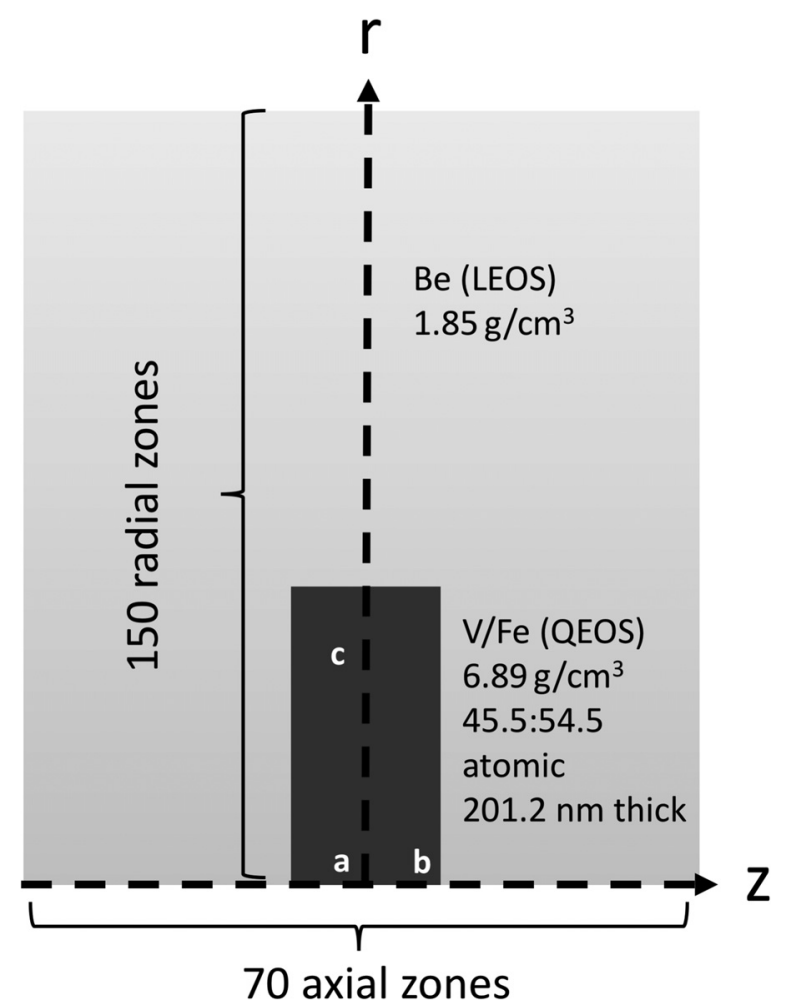

FIG. 3. Setup of 2D simulations in the HYDRA code. Be tamper with target in the center of the simulation. One half of the experiment is simulated with the expansion axis, $\mathbf{Z}$, being the axis of symmetry. Not presented in the figure is a large hemisphere of low density $\mathrm{He}, \rho=10^{-5} \mathrm{~g} / \mathrm{cm}^{3}$ with opacities set to 0 . This gas is used in order to mimic the vacuum environment in the experiment. The Be tamper is $5 \mu \mathrm{m}$ thick from each side of the buried layer. The buried layer itself is $2000 \AA$ thick. The picture is not to scale.

2. During the second phase, the laser is still being absorbed in the tamper material, the critical density surface has not reached the buried material. However, energy is conducted into the buried material mainly by two mechanisms: radiative transfer and

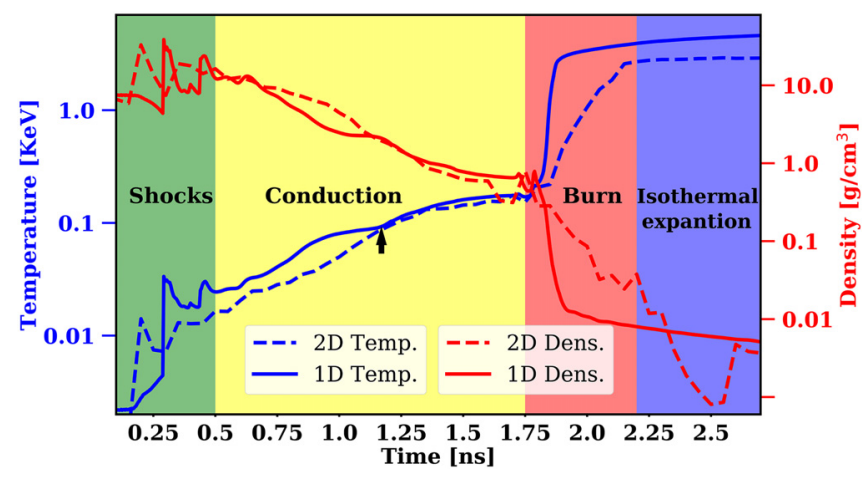

FIG. 4. Temperature and density plots of the buried layer target at $R=0, Z=0$ (point $a$ in Fig. 3). Dashed lines are the 2D simulation results. Solid lines are the 1D simulation results. The different phases in the target's dynamics are color marked. The arrow in the figure marks the transition from radiation conduction dominated phase to electron conduction phase. 
electronic conduction. Radiative transfer is the dominant mechanism in the earlier times of this phase, $\mathrm{t} \lesssim 1.2 \mathrm{~ns}$ while electronic conduction is dominant in the later times.

3. The transition between the two mechanisms can is marked with an arrow at $\mathrm{t} \approx 1.2 \mathrm{~ns}$ in Fig. 4 . A large change in slope can be observed in the $1 \mathrm{D}$ results for both the temperature and the density. In the $2 \mathrm{D}$ simulation, the effect of radiative heating on the target is reduced, compared to the $1 \mathrm{D}$ one. The plasma parameters in both simulations are in good agreement during the later part of this phase and no significant radial expansion is observed during this phase of the experiment. Therefore, the difference at $\mathrm{t} \lesssim 1.2 \mathrm{~ns}$ can be attributed to the different atomic and radiation transfer models used by the two codes. However, once the laser absorption front gets closer to the buried target and the electronic conduction becomes dominant, the two models align again.

4. The third phase takes place when the laser critical surface reaches the buried layer. Since the buried layer is very thin, originally $2000 \AA$, the laser quickly burns through this layer. From this time on, the entire target is under-dense.

5. The $2 \mathrm{D}$ simulations show a significantly slower evolution of the plasma parameters during the burnthrough phase. This is due to the radial expansion of the target, which lowers the density and as a result lowers the laser absorption rate. The radial expansion only lasts until pressure equilibrium between the buried material and the surrounding beryllium is achieved. This expansion is in agreement with the $\sim 40 \%$ radial expansion found in the experimental results in Fig. 2. This effect cannot be simulated in 1D.

6. In the last phase, the target continues to expand axially with both temperature and density only slowly changing in time. In this phase, the $1 \mathrm{D}$ and $2 \mathrm{D}$ simulations show qualitatively similar behavior. However, since in the previous phase, the $2 \mathrm{D}$ simulations included a $\sim 40 \%$ radial expansion, not present in the $1 \mathrm{D}$ simulations, the density in these simulations is reduced by approximately by a factor of 2 . The energy loss due to this mechanical work (Pdv) during the expansion leads to an approximately $\times 2$ decrease in the terminal temperature of the target in the $2 \mathrm{D}$ simulations.

In the following paragraphs, the axial and radial uniformity are discussed, and the temperature profiles are presented to demonstrate the target uniformity. The density profiles, both radial and axial, show uniformity which is similar to the temperature uniformity presented in Figs. 5 and 6; therefore, they are not presented in the figures.

Figure 5 demonstrates the target axial uniformity during the experiment. In this figure, the temperature at the buried layer center and edge are compared. The center is point $a$ in Fig. 3 and the edge is point $b$. It can be seen that both the $1 \mathrm{D}$ and $2 \mathrm{D}$ simulations show high level of axial uniformity before the electron conduction becomes the dominant heating mechanism and after the laser burnthrough.

The 2D simulations show high level of radial uniformity as well. This is demonstrated in Fig. 6. Comparing the plasma conditions along the central line of the buried layer, points $a$ to $c$ in Fig. 3 show that the conditions are approximately uniform up to $R>100 \mu \mathrm{m}$. The original target radius is $125 \mu \mathrm{m}$. The plasma conditions show similar behavior radially and axially. Conditions are close to being uniform during most of the experimental phases with non-uniformity being the largest during the burnthrough phase (see Fig. 4).

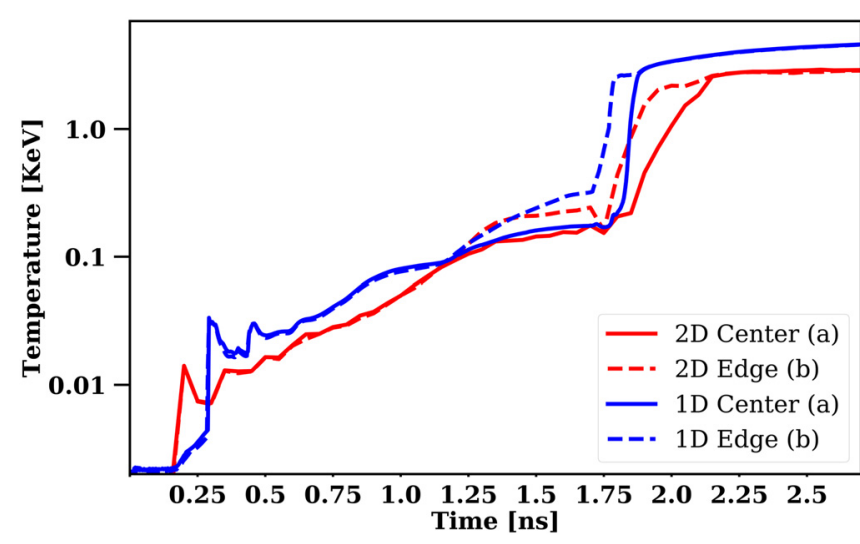

FIG. 5. Temperature plots of the buried layer target at the center, point $a$ in Fig. 3 and the edge point $b$ in Fig. 3. Dashed lines are the 2D simulation results. Solid lines are the $1 \mathrm{D}$ simulation results.

\section{EXPERIMENTAL ANALYSIS}

In Secs. V A-V C, a comparison of the simulations results with the experimental data is presented. The focus of the current work is the dynamic evolution of the target; hence, the analysis of the detailed spectra measurements, which were taken during the experiments, ${ }^{3}$ is beyond the scope of this work. Broad band and dynamic measurements are presented to support the dynamic picture presented in the previous sections. These simulations were performed using the laser pulse shape in the experiments, and all other experimental details are described in Sec. II.

\section{A. Target expansion imaging}

The first quantity to be compared is the time-dependent expansion of the buried layer itself. The expansion is taken from side-on imaging of the target as presented in Fig. 2. The experimental expansion is averaged over the radial direction.

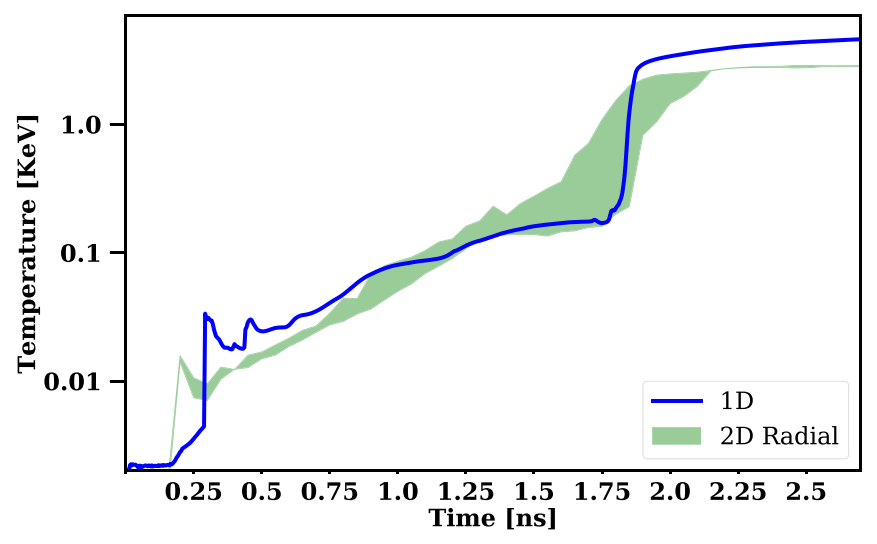

FIG. 6. Temperature plots of the buried layer target at the center along the radial direction, point $a$ to point $c$ in Fig. 3. The shaded green area represents the span of temperatures along this central line, in the $2 \mathrm{D}$ simulations. Solid blue line is the $1 \mathrm{D}$ simulation results. The largest non-uniformities are found during the burnthrough phase. 


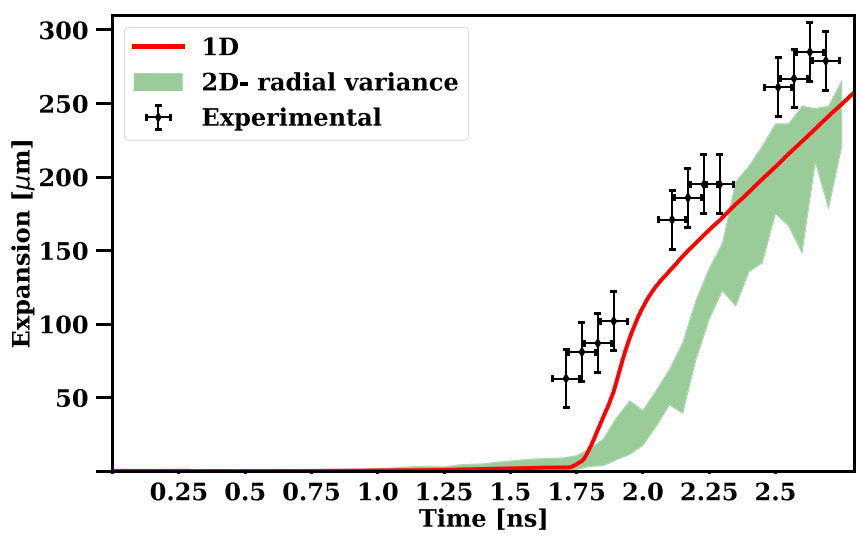

FIG. 7. Axial expansion of the buried layer target. Red-The 1D results and green-2D results. The shadowed region represents the variance in in the axial expansion along the radial direction. Black diamonds-experimental data with instrumental error.

An agreement is found in the target expansion dynamics between the simulations and the experimental data. Simulations still show a timing difference in the onset of the target expansion of approximately $0.1 \mathrm{~ns}$. However, both the expansion dynamics and the expansion sizes are in agreement between the experimental data and the simulations. The absolute timing of the gated imaging was estimated to have an error of $\sim 0.2 \mathrm{~ns}$. The results presented in Fig. 7 were shifted to best fit the simulated data within this error. The timing ( $\mathrm{x}$ axis) error bars in the figure represent the relative error between frames. The $2 \mathrm{D}$ results presented in Fig. 7 are the expansion of the pure buried layer material expansion only. An additional layer of mixed Be-target materials is found in the simulations. Accounting for some of the mixed material region can lead to a better agreement between the $2 \mathrm{D}$ simulations and the experimental data. However, since the mixed material does not have a clear boundary layer, it is not presented in this figure. The effect of the mixed material region is observed in spectral results presented in Sec. V B.

The hydrodynamic parameters used in Fig.7 are also the parameters that best fit the experimental data. The analysis of the parameters choice is further explained in Sec. VI. However, no other combination of hydro parameters and experimental uncertainty gave a better fit of the experimental and simulation results.

\section{B. Time-dependent $x$-ray emission-DANTE}

A second time-dependent measurement taken during the experiments was the broad band $\mathrm{x}$-ray emission measured by the DANTE $\mathrm{x}$ ray diodes array. ${ }^{6}$ As can be seen in Fig. 1, the laser spot covers an area significantly larger than the area containing the buried layer itself. A non-negligible radiation flux, especially at early times of the experiment originates from this region. In order to simulate the emitted radiation using a $1 \mathrm{D}$ code, a simplified two cylinders model was used. The model is described in Fig. 8.

Two independent $1 \mathrm{D}$ slab geometry simulations were used to represent the two regions of the experiment, i.e., the region containing the buried layer and the region with Be only. Each 1D simulation results in the irradiance per surface area in each region. The emitting surface area of each region was taken from the experimental target configuration. However, the slab geometry assumption is not valid for the external Be only cylinder throughout the duration of the experiment. Since the Be is not tamped by any other material, it can expand freely into the vacuum. As shown in Sec. IV for the buried layer, this

\section{Model overview}

Axial configuration

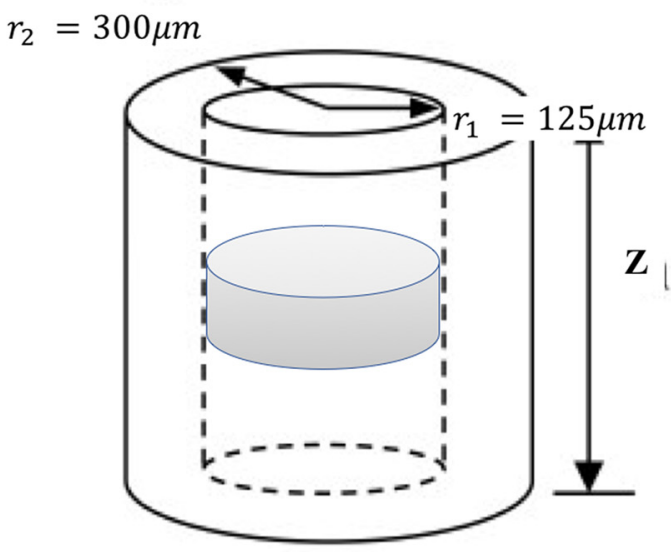

With Buried layer

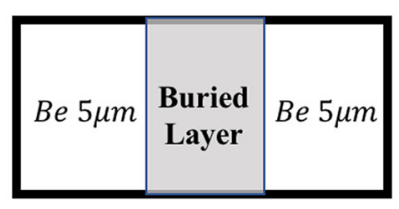

Be only region

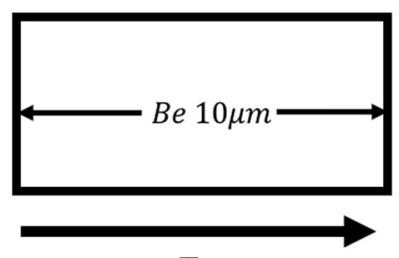

$\mathbf{Z}$

FIG. 8. Left: A schematic overview of the two cylinders geometry used in the $1 \mathrm{D}$ simulations. Right: the axial configurations of the two $1 \mathrm{D}$ simulations. The picture is not to scale. 
leads to an over estimation of the temperature. Since the Be contributes only a small portion of the total radiation, a factor of 0.5 was added to the emission of the Be only cylinder to account for this effect.

The results of the experimental and simulated voltage signals are presented in Fig. 9.

In order to obtain the simulated voltage signals, the simulated radiation coming out of the target was taken from the simulation. This flux was then multiplied by the DANTE solid angle and integrated over the detector response function, giving the simulated timedependent voltage signal.

It can be seen that the absolute peak signal values are in good agreement with both simulations. An inflection point can be observed in the two lower energy channels at approximately $1.2 \mathrm{~ns}$. This inflection point corresponds to the electron conduction heat front reaching the buried layer material (see Fig. 5). The largest discrepancies are found after the laser burnthrough at approximately $1.8 \mathrm{~ns}$. In the case of the 1D simulations, this discrepancy stems from the higher temperature predicted by the simulation. The higher temperature diminishes the population of the atomic levels contributing in the relevant spectral regimes. It can be seen that at later times, after approximately $2.2 \mathrm{~ns}$, the higher energy channels the $1 \mathrm{D}$ simulation predicted emission is higher than experimentally measured. The comparison between the $2 \mathrm{D}$ simulations and the experimental measurements suggest that the same is true in the $2 \mathrm{D}$ simulations as well. The $2 \mathrm{D}$ simulations are in agreement with the experimental values for a longer time but seem to fail at $t \approx 2.3 \mathrm{~ns}$. The qualitative behavior of the voltage signal at this later time is similar to the behavior observed earlier in time in the $1 \mathrm{D}$ simulations. Therefore, it is possible that this can be attributed to a more general three-dimensional dynamics in the experiments at later times which cannot be captured by the simulation. More direct measurements are needed to confirm this picture.
The signal timing observed in Fig. 9 is in agreement with the expansion results observed in Fig. 7. The experimental signal originating from the buried layer rises earlier than in the $1 \mathrm{D}$ simulation. The 2D simulations, however, seem to better match the signal rise time in the DANTE than could be expected from the expansion results presented in Fig. 7. This is caused by the earlier emission of mixed region of $\mathrm{Be}$ and $\mathrm{Fe} / \mathrm{V}$. However, as described in Sec. V A, the extant of this region could not be accounted for in the expansion results as no clear boundary of the mixed material could be defined in the $2 \mathrm{D}$ simulations results.

\section{Time-integrated $x$-ray emission-TGS}

The results presented in Secs. V A and V B were taken using a buried layer of mixed iron and vanadium. Recently, a new diagnostic capability was added to the experiments at the Omega laser facility. The new diagnostic consisted of a TGS coupled to a CCD camera. This diagnostic was designed and built at the Soreq research center. The specifications were similar to those presented in Ref. 7. The TGS gives a more spectrally resolved measurement of the emitted radiation. When coupled to a CCD camera, only a time-integrated measurement is achieved. The TGS's calibration is independent of the DANTE calibration, therefore giving another verification of the simulations' results. In the experiment presented in Fig. 10, a pure titanium buried target was used. All other target specifications were similar to the Fe-V targets described in Sec. II. Following the analysis of the Fe- $\mathrm{V}$ targets, 1D simulations were used in order to estimate the expected spectral flux. The simulation method was similar to the method described in Sec. VB. These simulations were used for the design of the grating and for analyzing the experimental results.
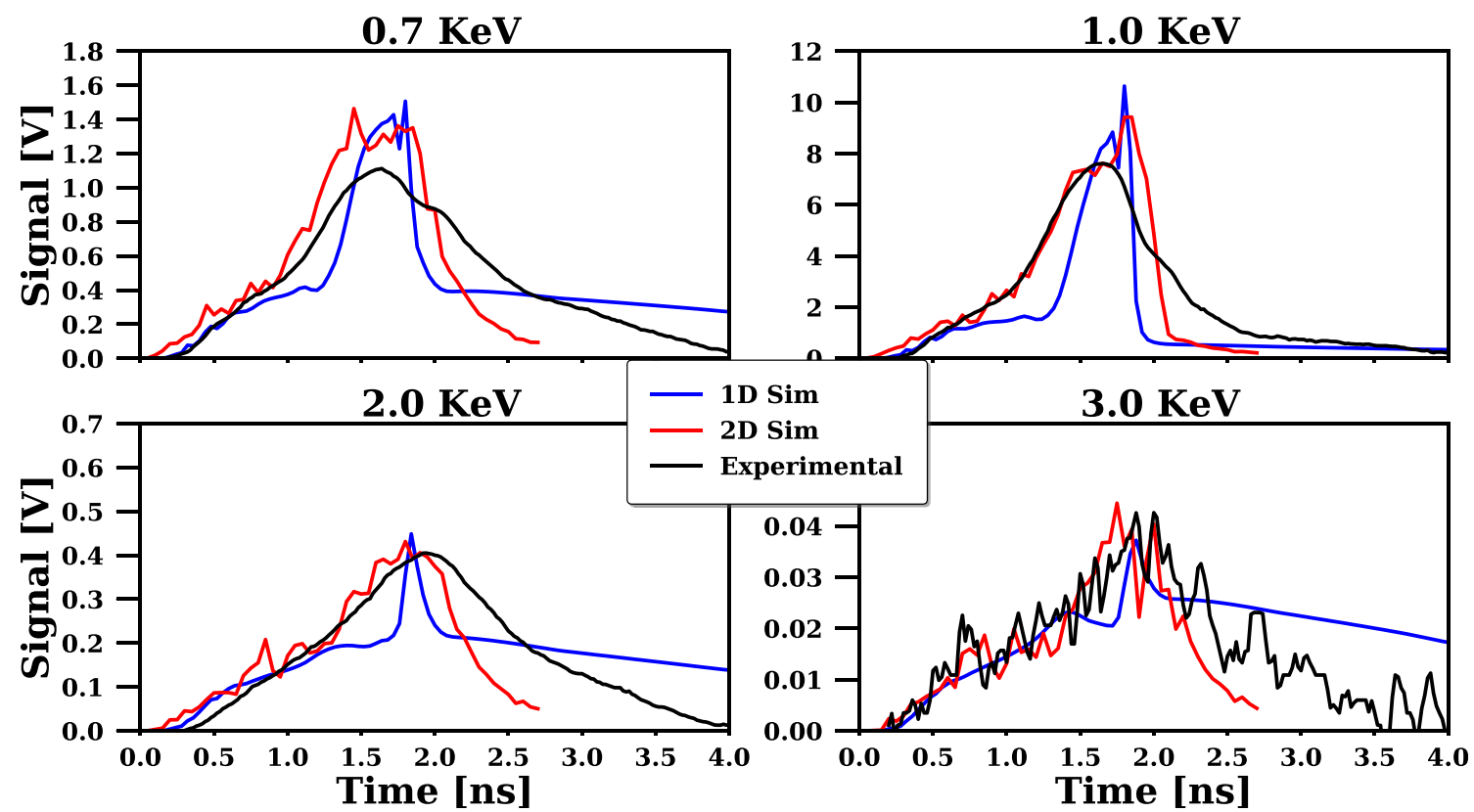

FIG. 9. Voltage signal of four DANTE channels, and the energy in the title of each plot is the central photon energy of the channel. Black curves are the experimental results, red curves are the 2D simulations, and blue curves are the $1 \mathrm{D}$ simulations. 


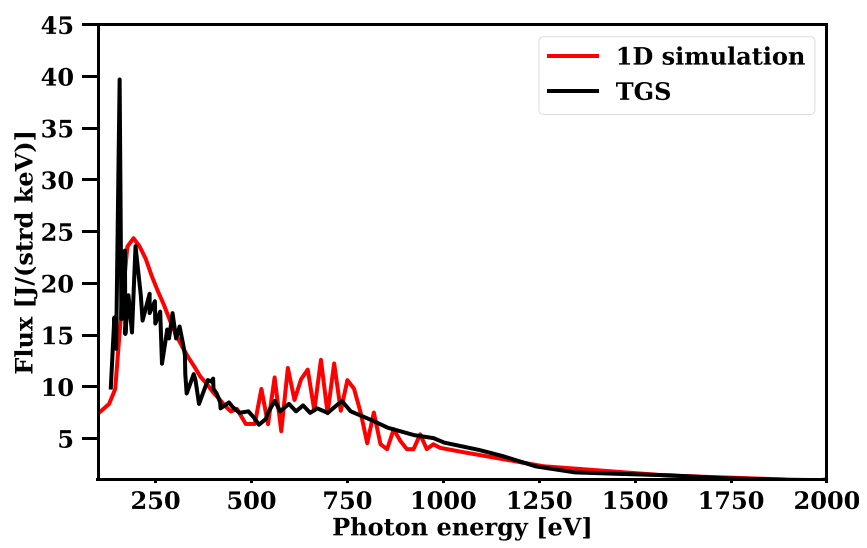

FIG. 10. Time-integrated flux. Black-TGS measurements. Red-1D simulations.

\section{PARAMETERS OF RADIATION-HYDRODYNAMIC SIMULATIONS}

In Secs. IV and V, we have demonstrated that the hydrodynamic evolution of the buried layer target is one-dimensional in the axial direction during most of the experimental duration. Moreover, in the 2D simulation, even after a radial expansion is taking place, the target evolution returns to be mostly axial for an additional period of time thereafter. Therefore, the $1 \mathrm{D}$ simulations can be used to perform a sensitivity check of the results to the choice of parameters used in the radhydro simulations.

The electron flux limiter parameter has been the subject of many discussions in recent years. ${ }^{13}$ Different experiments have supported the use of different values for this parameter. Figure 11 shows the variations in target expansion for different choices of the flux limiter values. It can be seen that the choice of a restrictive, $\mathrm{f}=0.03$, flux limiter

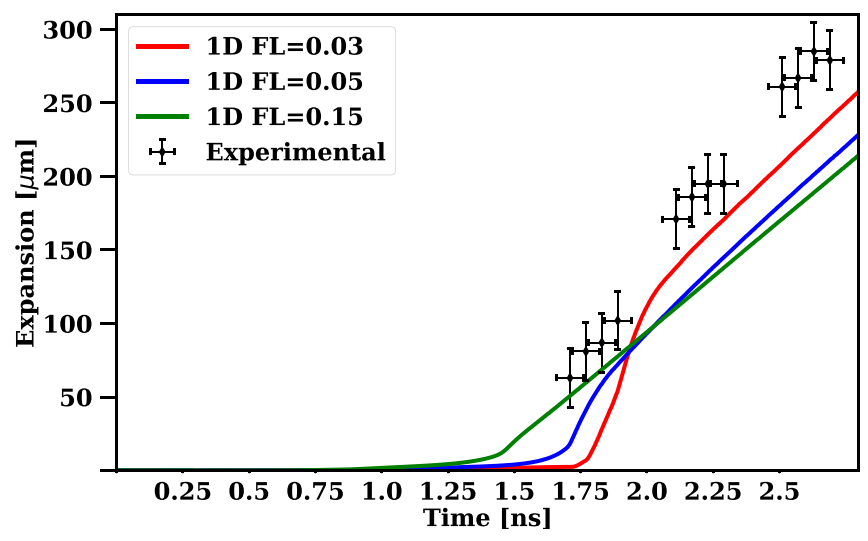

FIG. 11. Axial expansion of buried layer Fe-V target. Green-using flux limiter (FL) of 0.15 , blue-using flux limiter of 0.05 , and red-using a flux limiter of 0.03 . It can be seen that while the use of a larger value (less restrictive) flux limiter might be more fitting in the early stages of the target expansion, these flux limiters cannot capture the expansion velocity in later times. Overall, a restrictive value of 0.03 best fits the experimental expansion data. value best fits the experimental results. This value was also used in the 2D simulations.

A second hydrodynamic parameter used in the simulations is the fraction of resonant absorption of the laser energy which takes place near the critical density layer. ${ }^{14}$ Several combinations for the choice of the electron flux limiter and resonant absorption were examined using the $1 \mathrm{D}$ simulation. While this parameter has a smaller effect on the simulation's results, the $1 \mathrm{D}$ sensitivity check shows that absorption at the critical surface is needed in order to match the experimental results. A minimum of $30 \%$ of the remaining laser energy at critical was found necessary. Using a higher value had a small effect on the simulation's results but did not yield a significantly better fit with the experimental data. Resonant absorption was not used in the 2D simulations. It is possible that the instabilities in the plasma flow contribute to better coupling of the laser energy to the plasma. The lack of resonant absorption can also account for the delay in the target expansion in the 2D simulations seen in Fig. 7 as the rate of energy absorption from the laser is slower.

\section{CONCLUSIONS}

In this work, we presented the hydrodynamic simulations of buried layer experiments performed at the Omega laser facility. It was found that the buried layer targets show a near one-dimensional dynamics during their irradiation by the laser. Four main hydrodynamic phases were identified in these experiments. There is an initial phase of shocks going through the target. In a second phase, radiative and electron conduction heat the target. During the earlier radiative heating part of the second phase, a maximum temperature of $\sim 90 \mathrm{eV}$ is achieved. In these two phases, the expansion is approximately $1 \mathrm{D}$ axial. The target conditions are uniform during these stages. Moreover, at the later part of the conduction phase, the target conditions are only slowly changing over time.

In the next phase, the laser ablation front reaches the buried layer, and the laser quickly burns through the target. The buried layer reaches an electron temperature of approximately $2 \mathrm{keV}$ and electron density of several $10^{20} \mathrm{~cm}^{-3}$ to a few $10^{21} \mathrm{~cm}^{-3}$. At this stage, the $1 \mathrm{D}$ axial expansion dynamic breaks and a radial expansion also takes place. This radial expansion is mostly due to the pressure difference between the low $\mathrm{Z}$ Be tamper and the medium $\mathrm{Z}$ buried layer. When the buried layer's temperature quickly increases, the pressure in the target becomes higher than in the surrounding $\mathrm{Be}$, in the radial direction. This causes the layer to expand in that direction. However, once a pressure balance is regained, the radial expansion is suspended and only the axial expansion continues. The radial expansion causes the burnthrough process to be slower in the $2 \mathrm{D}$ picture than in the $1 \mathrm{D}$ one. Moreover, this expansion reduces the final density in the 2D simulations, hence reducing the laser plasma coupling. The 1D simulations therefore yield higher temperatures and densities past the burnthrough phase. A similar behavior was observed in the experimental imaging as well. ${ }^{3}$ This part of dynamics cannot be demonstrated in the $1 \mathrm{D}$ simulations.

After the laser burns through the target, the target expands slowly, maintaining almost constant temperature. The experiments described in this work aimed to measure the plasma emissivity during this phase.

The dynamic picture presented in this paper permits a better analysis of the buried layer experiments. It also allows us to further 
explore the use of this platform in other plasma regimes and explore possible new applications of the platform. For example, performing measurements right before the laser burns through the target might enable measurements at higher densities and lower temperatures. Time-resolved spectral measurements at the conduction phase of the experiment can allow us to study the electron conduction mechanism.

\section{ACKNOWLEDGMENTS}

The authors would like to thank Howard Scott, Duane Liedahl, Robert Heeter, and all other members of the LLNL radiative properties teem for many useful discussions regarding this work.

This work was performed under the auspices of the U.S. Department of Energy by LLNS, LLC, under Contract No. DEAC52- 07NA27344.

\section{DATA AVAILABILITY}

The data that support the findings of this study are available from the corresponding author upon reasonable request.

\section{REFERENCES}

${ }^{1}$ H. K. Chung and R. W. Lee, "Applications of NLTE population kinetics," High Energy Dens. Phys. 5(1-2), 1-14 (2009).

${ }^{2}$ H. A. Scott and S. B. Hansen, "Advances in NLTE modeling for integrated simulations,” High Energy Dens. Phys. 6(1), 39-47 (2010).

${ }^{3}$ E. V. Marley, D. A. Liedahl, M. B. Schneider, R. F. Heeter, L. C. Jarrott, C. W. Mauche, G. E. Kemp, M. E. Foord, Y. Frank, K. Widmann, and J. Emig, "Using 1-shell $\mathrm{X}$-ray spectra to determine conditions of non-local thermal dynamic equilibrium plasmas,” Rev. Sci. Instrum. 89(10), 10F106 (2018).

${ }^{4}$ W. J. Gray, M. E. Foord, M. B. Schneider, M. A. Barrios, G. V. Brown, R. F. Heeter, L. C. Jarrott, D. A. Liedahl, E. V. Marley, C. W. Mauche, and K.
Widmann, "Investigation of the hydrodynamics and emission of a laser heated tamped high-z target," Phys. Plasmas 25(6), 062702 (2018).

${ }^{5}$ G. Pérez-Callejo, L. C. Jarrott, D. A. Liedahl, E. V. Marley, G. E. Kemp, R. F. Heeter, J. A. Emig, M. E. Foord, K. Widmann, J. Jaquez, H. Huang, S. J. Rose, J. S. Wark, and M. B. Schneider, "Laboratory measurements of geometrical effects in the x-ray emission of optically thick lines for ICF diagnostics," Phys. Plasmas 26(6), 063302 (2019)

${ }^{6}$ K. M. Campbell, F. A. Weber, E. L. Dewald, S. H. Glenzer, O. L. Landen, R. E. Turner, and P. A. Waide, "Omega DANTE soft X-ray power diagnostic component calibration at the national synchrotron light source," Rev. Sci. Instrum. 75(10), 3768-3771 (2004).

${ }^{7}$ Z. Shpilman, G. Hurvitz, L. Danon, T. Shussman, Y. Ehrlich, S. Maman, I. Levy, and M. Fraenkel, "A combined sinusoidal transmission grating spectrometer and $\mathrm{x}$-ray diode array diagnostics for time-resolved spectral measurements in laser plasma experiments," Rev. Sci. Instrum. 90(1), 013501 (2019).

${ }^{8}$ Y. Frank, E. Raicher, Y. Ehrlich, G. Hurvitz, Z. Shpilman, M. Fraenkel, A. Zigler, and Z. Henis, "Influence of atomic modeling on integrated simulations of laser-produced au plasmas,” Phys. Rev. E 92, 053111 (2015).

${ }^{9}$ Y. Frank, E. Louzon, P. Mandelbaum, and Z. Henis, "Semillac: A new hybrid atomic model of hot dense plasmas," High Energy Dens. Phys. 9(3), 594-600 (2013).

${ }^{10}$ Y. Frank, P. Mandelbaum, and Z. Henis, "Semillac II: A new model for spectral behavior of hot plasmas," High Energy Dens. Phys. 12, 27-34 (2014).

${ }^{11}$ M. M. Marinak, B. A. Remington, S. V. Weber, R. E. Tipton, S. W. Haan, K. S. Budil, O. L. Landen, J. D. Kilkenny, and R. Wallace, "Three-dimensional single mode Rayleigh-Taylor experiments on nova," Phys. Rev. Lett. 75, 3677-3680 (1995).

${ }^{12}$ L. Spitzer, Jr., Physics of Fully Ionized Gases (Interscience, New York, 1962), Vol. 5.

${ }^{13}$ M. D. Rosen, H. A. Scott, D. E. Hinkel, E. A. Williams, D. A. Callahan, R. P. J. Town, L. Divol, P. A. Michel, W. L. Kruer, L. J. Suter, R. A. London, J. A. Harte, and G. B. Zimmerman, "The role of a detailed configuration accounting (DCA) atomic physics package in explaining the energy balance in ignition-scale hohlraums," High Energy Dens. Phys. 7(3), 180-190 (2011).

${ }^{14} \mathrm{~W}$. Kruer, The Physics of Laser Plasma Interactions (CRC Press, 2019). 